\section{A Stability Bias in Human Memory}

\author{
Nate Kornell \\ Department of Psychology, Williams College, \\ Williamstown, MA, USA
}

\section{Definition}

Human memory is anything but stable: We constantly add knowledge to our memories as we learn and lose access to knowledge as we forget. Yet people often make judgments and predictions about their memories that do not reflect this instability. The term stability bias refers to the human tendency to act as though one's memory will remain stable in the future. For example, people fail to predict that they will learn from future study opportunities; they also fail to predict that they will forget in the future with the passage of time. The stability bias appears to be rooted in a failure to appreciate external influences on memory, coupled with a lack of sensitivity to how the conditions present during learning will differ from the conditions present during a test.

\section{Theoretical Background}

All memories are not created equal. Some memories feel strong, vivid, and familiar; others feel shakier and less reliable. People are generally confident in the first type of memory but unsure about the second. Behavior reflects this difference; for example, most people only volunteer to answer a question in class if they feel confident about their response.

The term metacognition refers to the process of making judgments about one's cognition and, frequently, about one's memory (Dunlosky and Bjork 2008). Metacognitive processes are used to distinguish accurate memories from inaccurate ones. A memory is only valuable to the degree that we can trust it, which makes metacognition vital in our day-to-day use of memory. Moreover, virtually all memory retrievals are associated with a feeling of certainty (or lack thereof). Thus, metacognition is a critical, and omnipresent, component of human memory.
Metacognitive judgments are often accurate. For 39 example, your memory of what you ate for breakfast 40 today is probably more accurate than your memory of 41 what you ate for breakfast on this date 11 years ago, and 42 it probably feels more accurate as well. It would be natural 43 to assume that metacognitive judgments are made on the 44 basis of the memory being judged - that is, that when 45 confidence is low, it is because a memory is weak. The 46 empirical evidence suggests otherwise. $\quad 47$

Instead of being made based on memories themselves, 48 metacognitive judgments appear to be made based on 49 inferences about those memories. For example, if an 50 answer comes to mind quickly and easily, people tend to 51 judge that they know that answer well. This inference is 52 usually correct. But it is an inference all the same, 53 and when conditions are created that reverse this relation- 54 ship - when answers that come to mind quickly are less 55 memorable - people give high judgment of learning ratings 56 to information that comes to mind quickly, not to infor- 57 mation that is highly memorable (Benjamin et al. 1998). 58

If metacognitive judgments are inferential, what is the 59 basis of the inferences? Koriat (1997) put forward a highly 60 influential framework that has successfully accounted for 61 a great deal of subsequent data. He proposed that three 62 categories of cues influence metacognitive judgments. 63 Intrinsic cues were defined as information intrinsic to the 64 information being judged (e.g., the semantic relatedness 65 of a question and its answer). Mnemonic cues were defined 66 as information related to the learner's experience (e.g., the 67 fluency with which an answer comes to mind). Extrinsic 68 cues were defined as information extrinsic to the learner 69 and the to-be-learned material (e.g., the number of times 70 an item was studied).

A second key distinction, related to Koriat's (1997) 72 framework, is between judgments based on direct 73 experience and judgments based on analytical processes 74 (Kelly and Jacoby 1996). Intrinsic cues and mnemonic 75 cues tend to elicit experience-based judgments. That is, 76 these cues (e.g., how easily one thinks of an answer) are 77 part of the learner's experience at the time of the 78 judgment. Metacognitive judgments are usually highly 79 sensitive to a person's current experience. Thus, experi- 80 ence-based judgments often occur automatically. 
Extrinsic cues, by contrast, tend to elicit more analytical belief-based judgments. For example, the number of times an item will be studied is not a salient part of the learner's experience while studying. Instead, responding to an extrinsic cue often requires applying one's beliefs about memory (e.g., I will do better on items I study more). Doing so does not tend to happen automatically. As a result, people regularly fail to make belief-based judgments, even when they should. Thus, people tend to be sensitive to experience-based cues but not belief-based cues.

It is important to be able to predict how future events will affect one's memory. For example, a student may need to predict the value of spending the rest of the day studying. Future events are extrinsic cues - they are external to the learner's current experience - and, as such, they require belief-based judgments. Thus, people should exhibit a stability bias: They should be relatively insensitive to the impact of future events on their memories.

\section{Important Scientific Research and Open Questions}

Koriat et al. (2004) investigated how sensitive people are to future forgetting. After studying a list of word pairs, their participants were asked to predict their likelihood of recalling the pairs on a cued-recall test (i.e., their ability to recall the second word in the pair when shown the first word). There were three groups of participants, who were told, respectively, that their test would take place immediately, a day later, or a week later.

Actual recall performance dropped off precipitously as the delay between study and test increased. Shockingly, predictions hardly changed at all. In other words, the participants demonstrated a stability bias: They acted as though they would remember just as much in a week as they would remember immediately. The predictions were highly sensitive to the degree of association between the pairs, which is an experience-based, intrinsic cue. But they were insensitive to retention interval, an extrinsic cue. In one extreme case, tests that would take place immediately and in one year elicited the same predictions.

A key change in the procedure greatly altered participants' predictions. When a single participant was told about all three retention intervals, their predictions became sensitive to retention interval. It appeared as though the participants believed that they would forget over time, but that they did not apply that belief in the first experiment. When they were told about all of the retention intervals, they began to apply belief-based judgments. Phrasing the question in terms of forgetting had a similar effect: Apparently, making the idea of forgetting salient was enough to make judgments sensitive to reten- 132 tion interval.

One potential implication of ignoring retention inter- 134 val is extreme overconfidence. People tend to be 135 overconfident in their memories in general. But when 136 someone is overconfident about an immediate test, and 137 is not sensitive to retention interval, their overconfidence 138 is destined to grow. For example, assume you have a 70\% 139 chance of recalling a fact from your textbook if you are 140 tested in $10 \mathrm{~min}$. If you are tested in a week, that chance 141 might decrease to $20 \%$. If you judge that you have an $80 \% 142$ chance of recalling the fact at either retention interval, you 143 will be overconfident immediately, but only by $10 \% 144$ points. A week later, you will be overconfident by $60 \% 145$ points. This increase in overconfidence with time has been 146 referred to as long-term overconfidence (Kornell 2010). $\quad 147$

The stability bias is not limited to forgetting. Kornell 148 and Bjork (2009) investigated predictions about another 149 seemingly obvious principle of memory, namely, that 150 people learn by studying. Their participants were told 151 that they would be allowed to study a list of word pairs 152 between one and four times. They were asked to predict 153 how they would do when they took a test on the pairs. The 154 predictions were almost entirely insensitive to the number 155 of study repetitions, again demonstrating a stability bias. 156 The stability bias did not go both ways; people recognized 157 the value of past studying, but underestimated the value of 158 future studying. Like with forgetting, when the concept of 159 learning was made salient, in a within-participants design, 160 the predictions became more sensitive. Unlike forgetting, 161 however, the predictions continued to underestimate the 162 value of studying. As a result, across a number of different 163 experiments, participants were overconfident in their cur- 164 rent knowledge, but simultaneously underconfident in 165 their learning ability.

One potential implication of undervaluing future 167 study opportunities is that people might underestimate 168 their own learning potential. For example, a student might 169 look at a set of challenging course materials and decide to 170 drop out of a class, assuming that he or she cannot learn all 171 of the material. If this student is underconfident in his or 172 her learning, he or she might be giving up in the face of 173 a challenge that could be overcome.

174

\section{Cross-References}

- Confidence Judgments in Learning

- Cued Recall

- Metacognition and Learning

- Metacognitive Learning: The Effect of Item-Specific

Experiences

$\checkmark$ Overconfidence
175

176 177 179 180 181 
- Self-confidence and Learning

183

184

185

186

187

188

189

190

191

192

193 Memorizing, and Forgetting

\section{References} and Language, 35, 157-175.
The Role of Stability in the Dynamics of Learning,

Benjamin, A. S., Bjork, R. A., \& Schwartz, B. L. (1998). The mismeasure of memory: When retrieval fluency is misleading as a metamnemonic index. Journal of Experimental Psychology: General, 127, 55-68.

Dunlosky, J., \& Bjork, R. A. (Eds.). (2008). A handbook of metamemory and memory. Hillsdale: Psychology Press.

Kelly, C. M., \& Jacoby, L. L. (1996). Adult egocentrism: Subjective experience versus analytic bases for judgment. Journal of Memory
Koriat, A. (1997). Monitoring one's own knowledge during study: A cue- 194 utilization approach to judgments of learning. Journal of Experimen- 195 tal Psychology: General, 126, 349-370.

Koriat, A., Bjork, R. A., Sheffer, L., \& Bar, S. K. (2004). Predicting one's 197 own forgetting: The role of experience-based and theory-based pro- 198 cesses. Journal of Experimental Psychology: General, 133, 643-656. 199

Kornell, N., \& Bjork, R. A. (2009). A stability bias in human memory: 200 Overestimating remembering and underestimating learning. Journal 201 of Experimental Psychology: General, 138, 449-468.

202

Kornell, N. (2010). Failing to predict future changes in memory: A 203 stability bias yields long-term overconfidence. In A. S. Benjamin 204 (Ed.), Successful Remembering and Successful Forgetting: A Festschrift 205 in Honor of Robert A. Bjork (pp. 365-386). New York, NY: Psychology 206 Press. 